\title{
Stress of Dying is not Suppressed by High-dose Morphine or by Dementia
}

\author{
Zeynel A Erkut', Tamira Klooker', Eric Endert ${ }^{2}$, Inge Huitinga' and Dick F Swaab*, \\ 'Netherlands Institute for Brain Research, Amsterdam, The Netherlands; ' Laboratory of Endocrinology, Department of Clinical Chemistry, \\ Academic Medical Center of University of Amsterdam, Amsterdam, The Netherlands
}

\begin{abstract}
Hypothalamo-pituitary-adrenal (HPA)-axis activation is a response of the organism to psychological and physical stress, resulting in elevated levels of glucocorticoids, mainly cortisol in humans. In our previous studies we found post-mortem blood and cerebrospinal fluid (CSF) cortisol levels to be up to 20-fold higher than in vivo levels. Since clinical observations point to similar strong elevations of cortisol in fatally ill patients, we suggested that the high post-mortem cortisol levels might be due to the stress during the process of dying. We hypothesized that if the cortisol rise during dying is due to the psychological stress of the impending death, then the rise in cortisol should be inversely proportional to the degree of dementia, and that high-dose morphine giving analgesia, sedation, and sleep would suppress this response. Therefore, we measured the cortisol levels by radioimmunoassay (RIA) in the post-mortem CSF of 85 Alzheimer patients and 52 controls. In addition, post-mortem serum cortisol of 17 subjects from the Alzheimer group and nine from the control group were measured. The Alzheimer patients were subdivided according to their degree of dementia, as scored on the Reisberg Scale, before their death. All groups were further analyzed for the effect of morphine treatment, as well as for the effects of the confounding factors like age, gender, time, and season of death. Alzheimer patients had significantly higher cortisol levels than controls, both in CSF (mean (nmol/ I) \pm SEM: $482 \pm 32$ vs $285 \pm 30$, respectively, $p<0.00$ I) and in serum $(2854 \pm 279$ vs I $533 \pm 395, p=0.0$ I I). Mean CSF cortisol level of the severely demented Alzheimer group was even significantly higher than that of mildly demented group (508 \pm 35 vs $225 \pm 65$, $p=0.024)$ and controls $(p<0.00 \mathrm{I})$. Cortisol levels correlated positively with the degree of dementia in the Alzheimer group $(r=0.236$, $p=0.035)$. High-dose morphine did not cause a suppression of cortisol rise, neither in controls nor in Alzheimer patients. Our results indicate that the extreme elevations of cortisol levels during dying are rather due to the organic stress of the organism than to psychological stress of the patient, and is not suppressed by high-dose morphine.

Neuropsychopharmacology (2004) 29, I52-157, advance online publication, 03 September 2003; doi: I 0. I038/sj.npp. I 300299
\end{abstract}

Keywords: stress; dying; cortisol; human; cerebrospinal fluid; post mortem; hypothalamo-pituitary-adrenal axis; morphine; dementia

\section{INTRODUCTION}

Post-mortem cerebrospinal fluid (CSF) cortisol levels in control subjects, Alzheimer patients (Swaab et al, 1994), and multiple sclerosis patients (Erkut et al, 2002) are some 20fold higher than the levels found in in vivo lumbar puncture CSF samples (Erkut et al, 2002; Devinsky et al, 1993; Wood, 1982; Uete et al, 1970). These observations are in line with the studies reporting up to 5 to 15 -fold increased blood cortisol levels of critically ill-patients, such as patients with septic shock or massive bleeding (Uete et al, 1970; Sandberg et al, 1956; Jurney et al, 1987; Schroeder et al, 2001; Lamberts et al, 1997; Aygen et al, 1997). Since a positive

*Correspondence: Dr Dick F Swaab, Netherlands Institute for Brain Research, Meibergdreef 33, I 105 AZ Amsterdam, The Netherlands, Tel: +3I 20 5665500, Fax: + 31206961006 ,

E-mail: d.swaab@nih.knaw.nl

Received 10 March 2003; revised 20 July 2003; accepted 29 July 2003 Online publication: I August 2003 at http://www.acnp.org/citations/ Npp080 I0303098/default.pdf correlation was observed between the degree of elevation of endogenous cortisol levels and the survival rate in critical disease conditions, the strong increase in endogenous cortisol is presumed to be a protective reflex of the organism against a fatal threat (Schroeder et al, 2001; Lamberts et al, 1997; Aygen et al, 1997; Reincke et al, 1993; Garcia Garcia et al, 2001; Shimada et al, 2000; Bollaert et al, 1998). High-dose glucocorticoids are, therefore, often used as supportive medication for critically ill patients in intensive care units (Shimada et al, 2000; Bollaert et al, 1998). It is not clear at present whether the drive behind this rise in endogenous cortisol is the psychological stress of the life-threatening condition or the physical stress that the organism experiences due to deterioration of biological functions.

Cortisol is the major glucocorticoid in humans, maintaining the stress reaction of the body to all kinds of physical and psychological discomfort. Increase in cortisol secretion can take place very quickly, within minutes in acute stress conditions, and can stay at high levels for long 
periods, sometimes days, months, and even years in chronic disease conditions. The extent of cortisol rise to a stressor can show great variations between different subjects in similar conditions. Nevertheless, the ability of the organism to respond to stressful conditions by regulating its cortisol levels is seen as a sign of an effective defence system to protect its homeostasis.

Stress during critical disease condition can be subdivided into three components. Clinically, the most evident one is the stress coming from the physical discomfort that the patient experiences, such as pain and respiratory distress. The second component, the psychological stress which is mostly expressed as anxiety of the patient from being ill and suffering from the consequences of this condition, adds to the stress of the physical discomfort. The psychological stress can be extended to the stress coming from being aware of the fact that their condition can result in death. The patient can experience this stress even in the absence of severe pain or other physical discomfort. A third component is the stress that the 'organism' experiences because of organ and system failures resulting in deterioration of body functions, leading to nonreversible destruction of homeostasis and eventually to death. This component of stress, which we call the organic (physical) component of stress of death, is a basic response of all organisms, and would not necessarily require the presence of the kind of consciousness or awareness that we usually refer to as 'higher cortical functions'.

We hypothesized that if the extent of stress reaction during the last pre-mortem periods (moribund stage) would be predominantly due to the psychological agony coming from the idea of dying, the cortisol rise during this stage would be smaller in severely demented Alzheimer patients when compared to that of mildly demented patients or nondemented controls. We also hypothesized that administration of high-dose morphine during the last pre-mortem period would suppress the cortisol rise induced by physical discomfort as well as by psychological stress.

\section{SUBJECTS, MATERIALS, AND METHODS}

Cortisol levels in post-mortem CSF of 85 Alzheimer patients and 52 nondemented controls were measured by radioimmunoassay (RIA). Of the 26 of these subjects (17 of the Alzheimer and nine of the control subjects), post-mortem plasma was also available and cortisol levels were measured in this compartment, too. Postmortem CSF and blood were collected in the framework of the Netherlands Brain Bank (NBB, coordinator Dr R Ravid), from patients from whom a written consent for a brain autopsy and use of the material and clinical information for research purposes had been obtained. Subjects donating CNS material to the NBB are from a broad spectrum of age, health state, and living conditions. For example, Alzheimer patients and age-matched controls used for the current study come from nursery houses, homes for the elderly, university hospitals, or were subjects living on their own with regular medical and nursary care. The well-established family-doctor system of the Netherlands supplies good and reliable information on medical state and medications of the subjects who were living on their own. The medical files from the hospitals and the family doctors were also available to the NBB. Blood and ventricular CSF, obtained during autopsy, were immediately centrifuged and the supernatants were stored at $-80^{\circ} \mathrm{C}$ in $250-1000 \mu \mathrm{l}$ aliquots until used. Exclusion criteria for all subjects used in this study were pre-mortem corticosteroid treatment, sepsis, and massive hemorrhage. Subjects with Parkinson's changes were excluded on the basis of the neuropathological examination of the substantia nigra.

The diagnosis 'probable Alzheimer's disease' was made during life according to the NINCDS-ADRDA criteria (McKhann et al, 1984). A global deterioration score of 6-7, according to the Reisberg Scale, was designated as 'severely demented' $(n=73)$ and of $4-5$ as 'mildly demented' $(n=12)$ (Reisberg et al, 1982). The controls were not demented and did not have a primary neurological or psychiatric disease. The brains of all subjects were neuropathologically investigated in a systematic way, following the guidelines of the US National Institute on Aging for the definitive diagnosis of Alzheimer disease. The criteria for the extent of the neuropathological lesions were staged between 0 and VI according to Braak and Braak (1991) in 51 of the controls and 78 of the Alzheimer patients. The subjects were subdivided into three groups of severely demented Alzheimer patients, mildly demented Alzheimer patients, and controls according to the Reisberg Scale. These groups were further subdivided according to whether they were under high-dose morphine treatment or not during the last 2 weeks of life. In total, 19 of the controls, four of the mildly demented, and 50 of the severely demented Alzheimer patients were treated with morphine. Administered dose of morphine was minimum $15 \mathrm{mg}$ in $24 \mathrm{~h}$ for the last pre-mortem day (range $15-1000 \mathrm{mg} /$ day), and the cumulative doses for the last 3 days were between 60 and $1000 \mathrm{mg}$. Medical information from the files of patients revealed that all subjects, died either in a health and care facility or at home, received medical care for pain and physical discomfort.

Information on possible confounding factors, such as clock-time of death, month of death, post-mortem delay (PMD), storage period of the post-mortem material, sex, age, and $\mathrm{pH}$ of the CSF (the latter as a measure of agonal state at death, Ravid et al, 1992), was obtained from the database of the NBB, and the association with cortisol levels was investigated by ANOVA. Also the age of disease onset, severity of dementia according to the Reisberg Scale, severity of neuropathological $\mathrm{AD}$ changes according to Braak and Braak, ApoE alleles of the patients, and brain weight (brw) were tested for their associations with the cortisol levels.

Total cortisol was measured in CSF and serum by competitive immunoassay (DPC, USA) with an analytical sensitivity of $0.2 \mathrm{mg} / \mathrm{dl}(5.5 \mathrm{nmol} / \mathrm{l})$. Interassay variation was between 4 and $6 \%$. The statistical differences between groups were tested by one-way and multiple-way ANOVA with post hoc test and by two-tailed Student's $t$-test (with Levene's test for equality of variances). Correlations were tested by Pearson's test. A $p$-value of $<0.05$ was taken as significant. 


\section{RESULTS}

Alzheimer patients $(n=85)$ had a significantly higher mean cortisol level than controls $(n=52)$, both in the CSF (mean (nmol/l) \pm SEM: $482 \pm 32$ vs $285 \pm 30$, respectively, $p<0.001)$ and in serum $(n=17$ and 9 ; mean \pm SEM: $2854 \pm 279$ vs $1533 \pm 395$, respectively, $p=0.011$; Figure 1a). The severity of dementia was positively correlated to the CSF cortisol levels. Severely demented Alzheimer patients had significantly higher mean cortisol in their CSF when compared to mildly demented patients $(325 \pm 65$ vs $508 \pm 35, p=0.048)$ and to the controls $(p<0.001$; Figure 1b). Also the Reisberg Scales of Alzheimer patients were positively correlated to CSF cortisol levels $(r=0.236, p=0.035)$. Neuropathological scaling according to Braak, on the other hand, did not correlate with the cortisol levels, neither in the Alzheimer patients nor in the control groups $(0.2<p<0.6)$. There was no difference of mean CSF cortisol between mildly demented Alzheimer patients and controls $(p>0.5$; Figure $1 \mathrm{~b})$. There were no correlations between the ApoE alleles of the subjects and the cortisol levels in either the Alzheimer or the control group $(p>0.3)$.

Morphine administration was not associated with a significant change in CSF cortisol levels, neither in controls $(p=0.519)$ nor in the Alzheimer group $(p=0.167)$. Also no effect of morphine on cortisol was observed in relation to the severity of dementia ( $p>0.4$ in mildly and severely demented Alzheimer groups; Figure 2). We did not observe a correlation between the daily or cumulative doses of morphine and cortisol levels $(p>0.1)$.

One-way ANOVA indicated a significant impact of gender on cortisol levels in both groups. When analyzed in subgroups divided according to disease, gender, and morphine treatment (eight subgroups), there was a significant difference between the groups by one-way ANOVA $(p<0.001)$ and by post hoc tests. In general, in both Alzheimer and control groups, female patients had higher CSF cortisol levels compared to the male patients $(525 \pm 40$ vs $372 \pm 49, p=0.033$ in the Alzheimer group and $337 \pm 43$ vs $213 \pm 37, p=0.042$ in the control group). This sex difference in cortisol levels was more prominent in nonmorphine treated patients, both in Alzheimer ( $257 \pm 64$ vs $502 \pm 66, p=0.028)$ and control $(220 \pm 47$ vs $375 \pm 63$, $p=0.06)$ groups. When analyzed separately, the mean age of female patients was significantly higher than that of male patients in the Alzheimer group $(n=61$ and 24, mean \pm SEM: $82.2 \pm 1.2$ and $73.6 \pm 2.5$ respectively), but not in the control group ( $n=30$ and 22 , mean \pm SEM: $76.9 \pm 2.4$, and $75.6 \pm 2.8$, respectively). ANOVA revealed, however, no significant effect of age of the different genders on cortisol levels in Alzheimer and control groups.

Although in both Alzheimer patients and controls, winter was associated with rather lower levels of cortisol when compared to autumn and summer, season of death did reveal a significant effect only in the Alzheimer group, between the patients who died in winter and autumn $(p=0.025)$. ANOVA did not imply a further significant effect of season of death on cortisol in any of the groups. Another confounding factor, the clock-time of death (analyzed in four periods of day as 2300-0500, 0500-1100, $1100-1700$, and $1700-2300 \mathrm{~h}$ by one-way ANOVA), did not
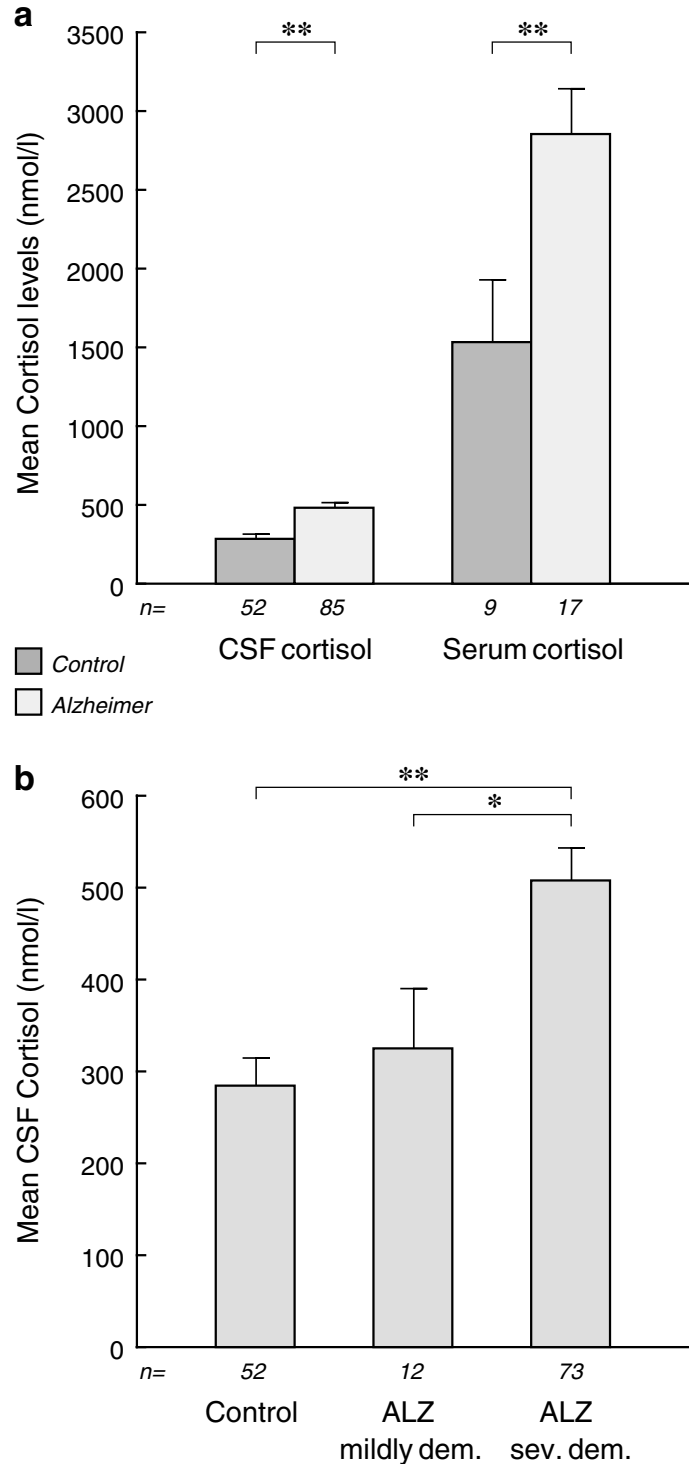

Figure I (a) Cortisol levels in the post-mortem serum and CSF of Alzheimer patients and controls. Cortisol levels of Alzheimer patients were significantly higher than those of controls, in both serum and CSF $(p=0.0 \mathrm{II}$ and $p<0.00 \mathrm{I}$, respectively). (b) CSF cortisol levels in relation to the presence and degree of dementia. Severely demented patients (Reisberg Scale $6-7, n=73$ ) had significantly higher cortisol level than mildly demented patients (Reisberg Scale $4-5, n=12)(p=0.048)$ and nondemented controls $(p<0.00 \mathrm{I})$. Note that cortisol levels of mildly demented Alzheimer patients did not significantly differ from those of controls $(p>0.2)$.

affect the cortisol levels in the Alzheimer or in the control group.

The mean PMD was significantly shorter in the Alzheimer group in comparison to controls $(263 \pm 9 v s 427 \pm 28 \mathrm{~min}$, $p<0.001)$. However, there was no correlation between PMD and cortisol neither in Alzheimer patients nor in the control groups $(r<0.2, p>0.3)$. Therefore, this difference in PMD will not have influenced our results. The $\mathrm{pH}$ of CSF was not different between the groups, and did not correlate with cortisol levels. When subjects were tested either altogether or in subgroups, storage time of CSF did not correlate with cortisol levels ( $p$ always $>0.3$ ). Age did not correlate with 


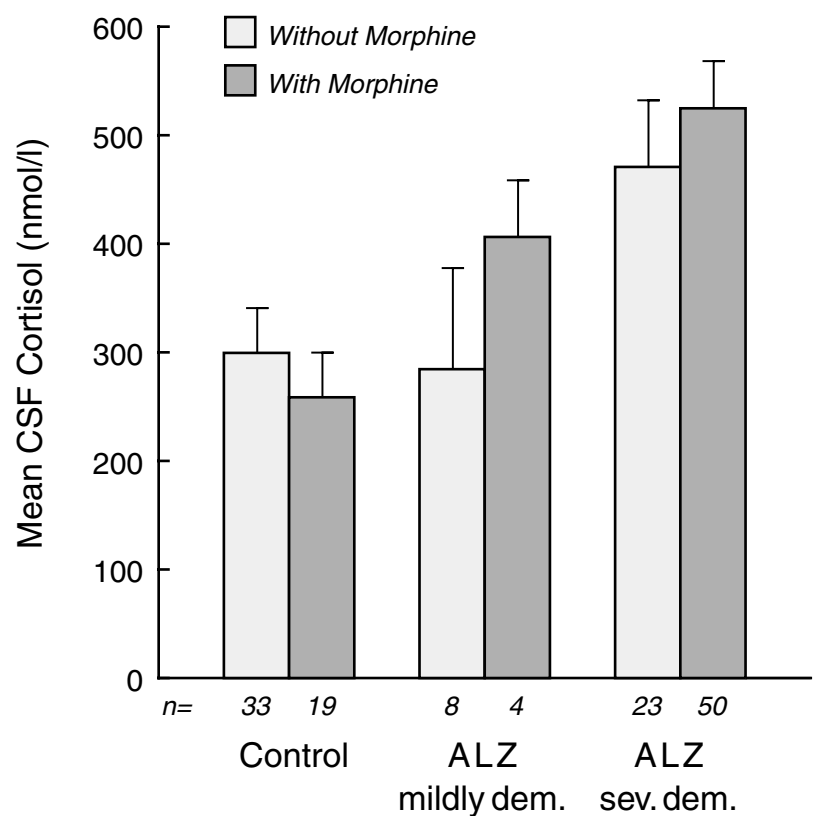

Figure 2 Effect of pre-mortem morphine on post-mortem CSF cortisol levels in controls and Alzheimer patients with severe and mild dementia. Note that morphine did not associate with a significant change of cortisol level in neither of the groups.

the cortisol levels either in Alzheimer or control groups, and did not show any effect on cortisol levels according to ANOVA ( $p$ always $>0.1$ ).

The mean brain weight of Alzheimer patients was significantly lower than that of controls $(1098 \pm 15 v s$ $1275 \pm 20 \mathrm{~g}$ respectively, $p<0.001)$. Also, when tested separately, the brain weight of both severely demented and mildly demented Alzheimer patients was lower than that of controls $(p<0.001$ and $p=0.035$, respectively). Moreover, the brain weight of severely demented patients was lower than that of mildly demented patients $(1086 \pm 15$ vs $1171 \pm 47$ respectively, $p=0.040$ ).

The cortisol levels of blood and CSF strongly correlated with each other in both groups $(n=9, r=0.748, p=0.020$ in controls and $n=17, r=0.794, p<0.001$ in the Alzheimer group; Figure 3). The blood/CSF cortisol ratio was similar in control and Alzheimer groups $(4.71 \pm 0.86$ vs $5.07 \pm 0.27$, respectively, $p>0.6$ ), indicating that serum cortisol is represented in similar ratios in the CSF of the subjects in all groups.

\section{DISCUSSION}

The post-mortem CSF cortisol levels in the current study were some 15- to 20-fold higher than the in vivo lumbar puncture levels reported in the literature, for both Alzheimer patients and controls, which confirms our earlier studies (Swaab et al, 1994; Erkut et al, 2002). In the present study, post-mortem CSF cortisol levels highly correlated with the post-mortem serum cortisol levels (Figure 3) with a similar ratio between the two compartments for both controls and Alzheimer patients, indicating that the blood-CSF barrier for this hormone is intact in Alzheimer's disease. Unbound (free) serum cortisol, which constitutes $10-20 \%$ of total serum cortisol, is shared by other body fluid

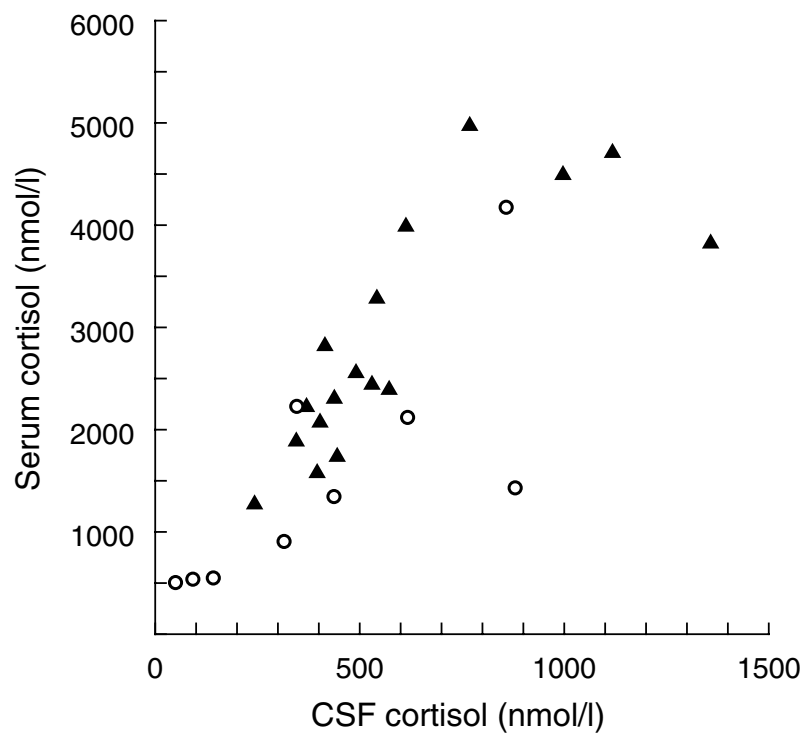

\section{- Alz o Control}

Figure 3 Correlation between post-mortem serum and CSF cortisol levels in a randomly selected group of Alzheimer's and control subjects. The correlation was significant both within the control $(r=0.748$, $p=0.020)$ and Alzheimer $(r=0.794, p<0.00 \mathrm{I})$ groups, and also when all subjects were pooled $(r=0.890, p<0.00 \mathrm{I})$.

compartments, including the CSF (Uete et al, 1970). The strong correlation between serum and CSF cortisol in our study with similar blood/CSF ratios indicates that the integrity of the blood-CSF barrier and the relationship between the two compartments is preserved during and after the death of the subjects, and that the high postmortem CSF cortisol is a reflection of high free serum cortisol levels.

We have observed similarly high post-mortem CSF cortisol levels before in controls, Alzheimer patients, and MS patients (Swaab et al, 1994; Erkut et al, 2002). The high levels of cortisol during the moribund stage were also observed by clinical researchers in different kinds of fatal conditions (Sandberg et al, 1956; Reincke et al, 1993; Schroeder et al, 2001). Since patients who cannot achieve a cortisol rise during critical health conditions due to adrenal insufficiency have significantly lower survival rates (Schroeder et al, 2001), the rise in endogenous cortisol in fatal conditions is thought to help the patient to survive this critical stage.

In order to see whether psychological stress is more important than the organic (physical) stress for the activation of the hypothalamo-pituitary-adrenal (HPA)-axis during death, we have investigated whether the degree of dementia was inversely correlated with the cortisol rise during the moribund period. We observed, on the contrary, that Alzheimer patients had an even more extreme rise in their cortisol levels during dying, showing a significant $70 \%$ higher mean levels of cortisol in the post-mortem CSF in comparison to controls, which confirms earlier results (Swaab et al, 1994). Moreover, severely demented patients had higher cortisol levels than mildly demented or nondemented patients. It is important to emphasize at this point the difference between the higher basal cortisol levels in demented patients, which is a 1.5 - to 2.5 -fold increase in 
Alzheimer patients in in vivo studies, and the cortisol rise during dying, which is much higher, up to 20 -fold, as observed in Alzheimer patients and in controls in our postmortem studies. The significant, but rather mild, elevation of basal cortisol in demented patients in vivo may come at least partly from a decreased inhibition of CRH neurons by the hippocampus, which is seriously affected by the Alzheimer process (Herman and Cullinan, 1997), but also partly from the psychological stress of being confused, lost, or isolated. Difficulty to understand the cause and course of their physical discomfort and pain may add to this stress. Many of these patients are already suffering for a long time from life-threatening health conditions, or are in the terminal stages of deadly diseases. Yet, the psychological stress of being in such conditions does not bring the cortisol rise to the extremely high levels seen in our post-mortem studies, until the death process really starts. Therefore, the up to 20 -fold increase of cortisol in post-mortem blood and CSF of demented patients cannot be explained by the stress coming from dementia. Our working hypothesis was that the cortisol levels of these patients would stay at the basal in vivo levels, that is, higher than the in vivo cortisol levels of the controls, but much lower than the post-mortem levels, if the psychological stress of dying was the main drive for the strong cortisol release. Our results showed, however, that a lower cognitive level and a decreased awareness of the subjects to the ongoing threat to their lives did not result in a decreased stress response during dying. Therefore, the elevation of cortisol during dying seems not to be due to the psychological stress of being in a critical life-threatening condition. This conclusion was supported by the data obtained from the subjects who were administered morphine. The rise of cortisol levels during death was not suppressed by high-dose morphine in either group. Morphine had no significant effect on cortisol levels, also when analyzed separately according to the severity of dementia or the gender of patients. The clinical indication, doses, and duration of high-dose morphine administration for the subjects in our study varied. Morphine was mostly given to quell the suffering from pain and discomfort, and against anxiety and restlessness (Burns et al, 1992; Turner et al, 1996, LeGrand et al, 2001). However, a number of subjects in our study received much higher doses (up to $1000 \mathrm{mg} /$ day), by which the level of consciousness of the patients, too, is changed. Morphine is shown to suppress cortisol in a number of other studies, not only in control subjects but also in psychologically or physically stressed patients (Zis et al, 1984; Palm et al, 1997; Pokela, 1993). Nevertheless, morphine-administered-moribund patients in our study, who, therefore, should not suffer from pain and physical discomfort, still had high levels of cortisol, indicating that physical discomfort and accompanying psychological distress did not play a major role in the cortisol rise during dying. As a result, our findings support the view that the extreme rise in cortisol during the moribund period levels is mainly a reaction of the organism to the deterioration of the biological functions, rather than due to the psychological stress of the patient from being in physical discomfort or in a critically ill condition.

One can argue that the level of cortisol rise as a result of organic stress during a fatal condition is related to the duration of the critical situation, the acceleration of shifting from 'critical state' to the 'process of dying', and the ability of the HPA-axis to respond to the biological stress, in order to reach its individual ceiling for cortisol level. The premortem clinical data of the subjects showed that the average duration in the critical state and the speed of deterioration of vital functions were similar in the two patient groups in our study. There were no sudden and unexpected deaths due to, for example, accidents, and no deaths as a result of massive bleeding or extreme infectious situations such as sepsis among the subjects studied. Therefore, there were no extreme health situations of the subjects during dying, which would cause the very strong cortisol response. The similar and reasonably low standard error of mean (SEM) values for cortisol in all groups supports this conclusion (Figure 1).

It must also be noted that cortisol levels in post-mortem CSF did not show a correlation with the $\mathrm{pH}$ of the CSF, neither in controls nor in Alzheimer patients. The $\mathrm{pH}$ of the CSF is related to the agony of the patient in relation with the respiratory distress during dying (Ravid et al, 1992). This supports, once again, that respiratory stress does not play a major role in the organic stress that leads to the strongly increased cortisol levels in the last stage of life.

Various in vivo studies report higher basal plasma and CSF cortisol levels in Alzheimer patients in comparison to controls (Masugi et al, 1989; Miller et al, 1998; Stanwick et al, 1998; Peskind et al, 2001), and in the severely demented patients in comparison to mildly demented ones (Masugi et al, 1989; Miller et al, 1998). These observations are fully in line with the results from the current postmortem study. Therefore, our results indicate that the increased HPA-axis responsiveness during death in Alzheimer patients is proportional to the increased basal HPAaxis activity levels that the patients had before the critical situation started (Masugi et al, 1989), and that the higher post-mortem cortisol levels of Alzheimer patients do not mean that they suffered more during dying.

In conclusion, the stress of death, which leads to extreme elevations in cortisol levels, is of an organic (physical) nature more than a psychological one, as it is not inhibited by increased level of dementia, and is not suppressed by high-dose morphine administration.

\section{ACKNOWLEDGEMENTS}

We thank W Hoogendijk, MD PhD and Dr E Fliers, MD, $\mathrm{PhD}$ for their comments and suggestions on the manuscript, and $\mathrm{H}$ Stoffels for the artwork.

\section{REFERENCES}

Aygen B, Inan M, Doganey M, Kelestimur F (1997). Adrenal functions in patients with sepsis. Exp Clin Endocrinol Diabetes 105: $182-186$.

Bollaert PE, Charpentier C, Levy B, Debouverie M, Audibert G, Larcan A (1998). Reversal of late septic shock with supraphysiologic doses of hydrocortisone. Crit Care Med 26: 645-650.

Braak H, Braak B (1991). Demonstration of amyloid deposits and neurofibrillary changes in whole brain sections. Brain Pathol 1: 213-216.

Burns AM, Shelly MP, Park GR (1992). The use of sedative agents in critically ill patients. Drugs 43: 507-515. 
Devinsky O, Emoto S, Nadi NS, Theodore WH (1993). Cerebrospinal fluid levels of neuropeptides, cortisol, and amino acids in patients with epilepsy. Epilepsia 34: 255-261.

Erkut ZA, Endert F, Huitinga I, Swaab DF (2002). Interleukin-6 and cortisol in post-mortem cerebrospinal fluid of multiple sclerosis patients and controls. Mult Scler 8: 229-236.

Garcia Garcia E, Milano Manso G, Lopez Siguero JP, Valls Moreno E, Calvo Macias C (2001). Adrenal function in children with sepsis and septic shock. (Funcion suprarrenal en ninos con sepsis y shock septico). An Esp Pediatr 54: 439-443.

Herman JP, Cullinan WE (1997). Neurocircuitry of stress: central control of the hypothalamo-pituitary-adrenocortical axis. Trends Neurosci 20: 78-84.

Jurney TH, Cockrell JL, Lindberg JS, Lamiell LM, Wade CE (1987). Spectrum of serum cortisol response to ACTH in ICU patients. Chest 92: 292-295.

Lamberts SWJ, Braining HA, de Jong FH (1997). Corticosteroid therapy in severe illness. Drug Ther 337: 1285-1292.

LeGrand SB, Tropiano P, Marx JD, Davis MP, Walsh D (2001). Dying at home: emergency medications for terminal symptoms. Am J Hosp Palliat Care 18: 421-423.

Masugi F, Ogihara T, Sakaguchi K, Otsuka A, Tsuchiya Y, Morimoto $S$ et al (1989). High plasma levels of cortisol in patients with senile dementia of the Alzheimer's type. Methods Find Exp Clin Pharmacol 11: 707-710.

McKhann G, Drachman D, Folstein M, Katzman R, Price D, Stadlan EM (1984). Clinical diagnosis of Alzheimer's disease: report of the NINCDS-ADRDA Work Group under the auspices of Department of Health and Human Services Task Force on Alzheimer's Disease. Neurology 34: 939-944.

Miller TP, Taylor J, Rogerson S, Mauricio M, Kennedy Q, Schatzberg A et al (1998). Cognitive and noncognitive symptoms in dementia patients: relationship to cortisol and dehydroepiandrosterone. Int Psychogeriatr 10: 85-96.

Palm S, Moening H, Maier C (1997). Effects of oral treatment with sustained release morphine tablets on hypothalamo-pituitaryadrenal axis. Methods Find Exp Clin Pharmacol 19: 269-273.

Peskind ER, Wilkinson CW, Petrie EC, Schellenberg GD, Raskind MA (2001). Increased CSF cortisol in AD is a function of APOE genotype. Neurology 56: 1094-1098.

Pokela ML (1993). Effect of opioid-induced analgesia on betaendorphin, cortisol and glucose response in neonates with cardiorespiratory problems. Biol Neonate 64: 360-367.
Ravid R, Van Zwieten EJ, Swaab DF (1992). Brain banking and the human hypothalamus-factors to match for, pitfalls and potentials. Prog Brain Res 93: 83-95.

Reincke M, Allolio B, Wurth G, Winkelmann W (1993). The hypothalamic-pituitary-adrenal axis in critical illness: response to dexamethasone and corticotropin-releasing hormone. J Clin Endocrinol Metab 77: 151-156.

Reisberg B, Ferris SH, Deleon MJ (1982). The global deterioration scale for assessment of primary degenerative dementia. Am J Psychiatry 139: 1136-1139.

Sandberg AA, Fik-Nes K, Migeon CJ, Samuels LT (1956). Metabolism of adrenal steroids in dying patients. J Clin Endoc Metab 16: 1001-1016.

Schroeder S, Wichers M, Klingmuller D, Schroeder S, Wichers M, Klingmuller D et al (2001). The hypothalamicpituitary-adrenal axis of patients with severe sepsis altered response to corticotropin-releasing hormone. Crit Care Med 29: 310-316.

Shimada H, Ochiai T, Okazumi S, Shimada H, Ochiai T, Okazumi S et al (2000). Clinical benefits of steroid therapy on surgical stress in patients with esophageal cancer. Surgery 128: 791-798.

Stanwick GR, Kirby M, Bruce I, Buggy F, Coen RF, Coakley D et al (1998). HPA-axis dysfunction in Alzheimer's disease: lack of association between longitudinal and cross-sectional findings. Am J Psychiatry 155: 286-289.

Swaab DF, Raadsheer FC, Endert F, Hofman MA, Kamphorst W, Ravid R (1994). Increased cortisol levels in aging and Alzheimer's disease in postmortem cerebrospinal fluid. $J$ Neuroendocrinol 94: 681-687.

Turner K, Chye R, Aggarwal G, Philip J, Skeels A, Lickiss JN (1996). Dignity in dying: a preliminary study of patients in the last three days of life. J Palliat Care 12: 7-13.

Uete T, Nishimura S, Oya $\mathrm{H}$, Shimomura $\mathrm{T}$, Tatebayashi $\mathrm{Y}$ (1970). Corticosteroid levels in blood and cerebrospinal fluid in various diseases. J Clin Endocrinol Metab 30: 208-214.

Wood JH (1982). Neuroendocrinology of cerebrospinal fluid: peptides, steroids, and other hormones. Neurosurgery 11: 293-305.

Zis AP, Haskett RF, Albala AA, Caroll BJ (1984). Morphine inhibits cortisol and stimulates prolactin secretion in man. Psychoneuroendocrinology 9: 423-427. 